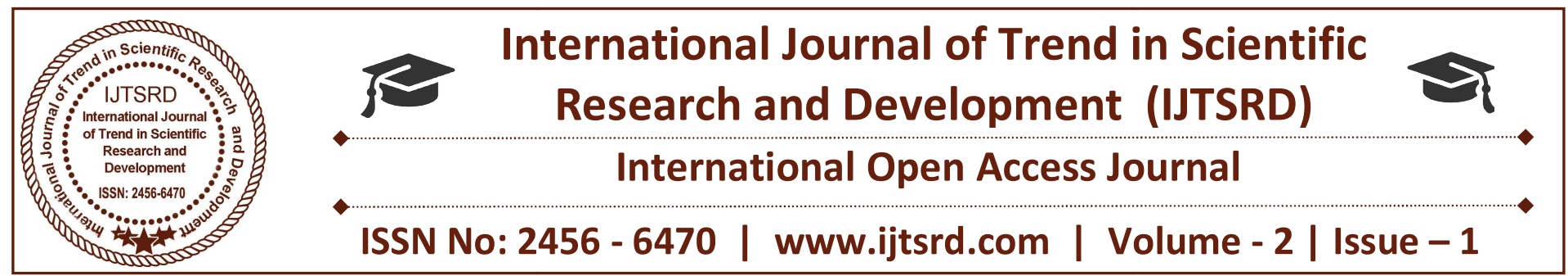

\title{
Optimizing Stress Parameters for Improving Lipid Accumulation in Heterotrophic Microalgae Cultivation for Biodiesel Production
}

\author{
Bhupendra Prasad, Jitendra Malviya, Supriya Maity \\ Department of Microbiology Career College Bhopal Govindpura BHEL, Bhopal (M.P.)
}

\begin{abstract}
In recent years, increasing consumption of conventional energy has caused serious concerns about energy security and environmental degradation. Microalgae are a diverse group of prokaryotic and eukaryotic photosynthetic microorganisms that grow rapidly due to their simple structure. It has long been considered as a promising alternative and renewable feedstock source for biofuels. It is $3^{\text {rd }}$ generation fuel, because as compared with terrestrial plants, microalgae have a high oil content and growth rate. In microalgae triacylglycerides (TAGs) generally served as energy storage that, once extracted, can be easily converted into biodiesel through trans-esterification reactions. Heterotrophic systems are more suitable for producing high cell densities of microalgae for accumulation of large quantities of lipids (triacylglycerols) which can be converted into biodiesel and its growth consumes simple, cheap, and available carbon sources (glucose, acetate, glycerol) that are commonly used by fermentation industries for other aims, it is predicted that adoption of this approach is an easy, effective commercial implementation of the biodiesel from microalgae strategy. Information concerning the composition of carbohydrates produced by microalgae is also crucial for future applications of microalgae feedstock.
\end{abstract}

Keywords: conventional, degradation, Microalgae, prokaryotic, eukaryotic, renewable, feedstock, triacylglycerides, trans-esterification, Heterotrophic, lipids, biodiesel, fermentation.

\section{INTRODUCTION}

Many technologies and sources of energy compulsorily replaced fossil fuels and contribute to the reduction of emissions of greenhouse gases associated with their use. Biofuels are particularly important as an alternative option for transportation fuels.[1] However, biofuels derived from terrestrial crops such as sugarcane, soybeans, maize, and rapeseed, among others, impose pressure on food markets, contribute to water scarcity and precipitate forest devastation. In this way, the sustainability of biofuels will depend on the development of viable, sustainable, second-generation technologies that do not appear to be yet commercially viable and cost effective too.[2]

Microalgae can generate diverse biofuels, which are mainly: biomethane produced by anaerobic digestion, biohydrogen by photobiological process, bioethanol by fermentation, liquid oil by thermal liquefaction and biodiesel. Microalgae have long been considered as a promising alternative and renewable feedstock source for biofuels also known as third generation fuels. Compared with terrestrial plants, microalgae have a high oil content and growth rate. Triacylglycerides (TAGs) generally serve as energy storage in microalgae that, once extracted, can be easily converted into biodiesel through trans esterification reactions.[5] Utilization of renewable energy for Sustainable production is increasingly understood that first generation biofuels, primarily produced from crops, weeds and food gradients and mostly produced by seeds are limited in their ability to achieve targets for biofuel production. The burning issued of climate change the mitigation and economic growth. Microalgae, has potential to offer a greatest opportunities in the longer term fuels requisition. The microalgae species and their advantage comparison with other available biodiesel feedstocks. For biodiesel 
production, cultivation, harvesting, and processing microalgae is very high in demand. The various aspects associated with the design of microalgae production units are described, giving an overview of the current state of development of algae cultivation systems (photo-bioreactors and open ponds). Other potential applications and products from microalgae are also presented such as for biological sequestration of $\mathrm{CO} 2$, wastewater treatment, in human health, as food additive, and for aquaculture. $[8,3]$ The main objective of the study to Isolation of pure strains_from mixed microalgae to optimizing stress parameters for improving lipid accumulation in mixed microalgae and microalgae isolates in heterotrophic conditions. Heterotrophic cultivation may allow large volume applications such as wastewater treatment combined, or separated, with production of biofuels. Heterotrophic cultivation can overcome the limitations faced in open pond and large-scale PBRs.[6]

\section{Material and Method}

\section{Sample Collection}

Collection of fresh water-borne microalgae inoculums from water bodies for analysis of fresh microalgae with their Biomass density, Chlorophyll, Lipid content and feed preparation for heterotrophic growth of algae. Fresh water needs to be added to raceway pond systems to compensate evaporation; water may also be used to cool some PBR designs.

\begin{tabular}{|l|l|c|}
\hline Culture & Treatment & Control \\
\hline Carbon source & $15 \mathrm{~g} / \mathrm{L}$ dextrose & - \\
\hline Other source & $1 \mathrm{X} \mathrm{DSW}$ & $1 \mathrm{X} \mathrm{DSW}$ \\
\hline
\end{tabular}

Table 1. Feed composition
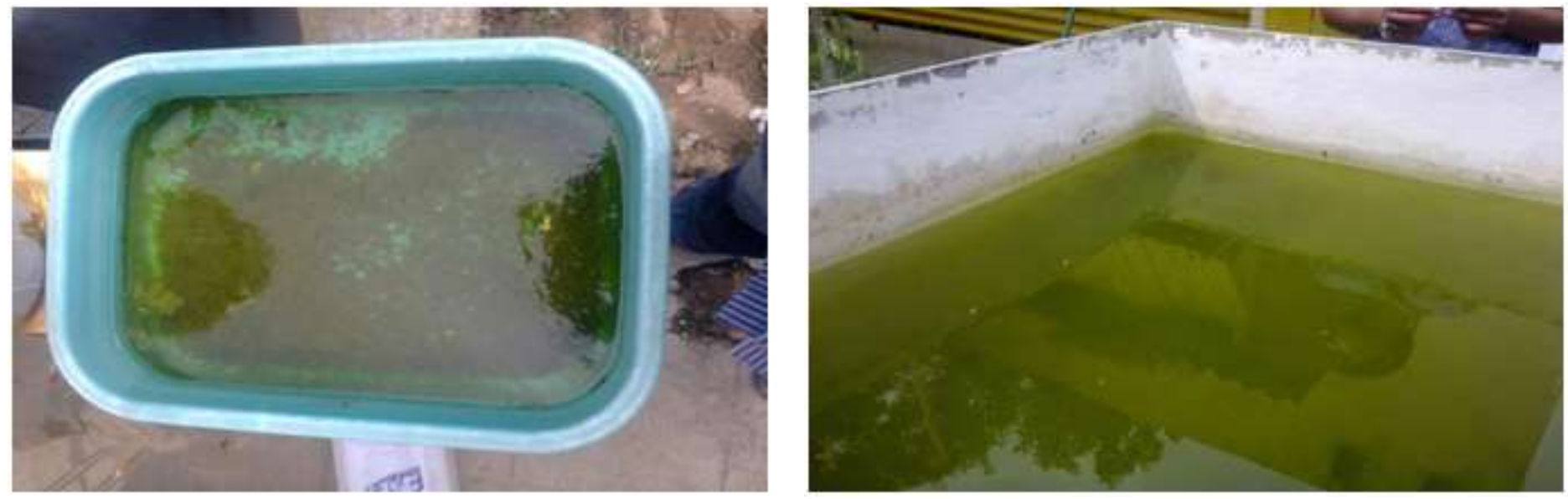

Fig.1. Collection and analysis of Microalgae 

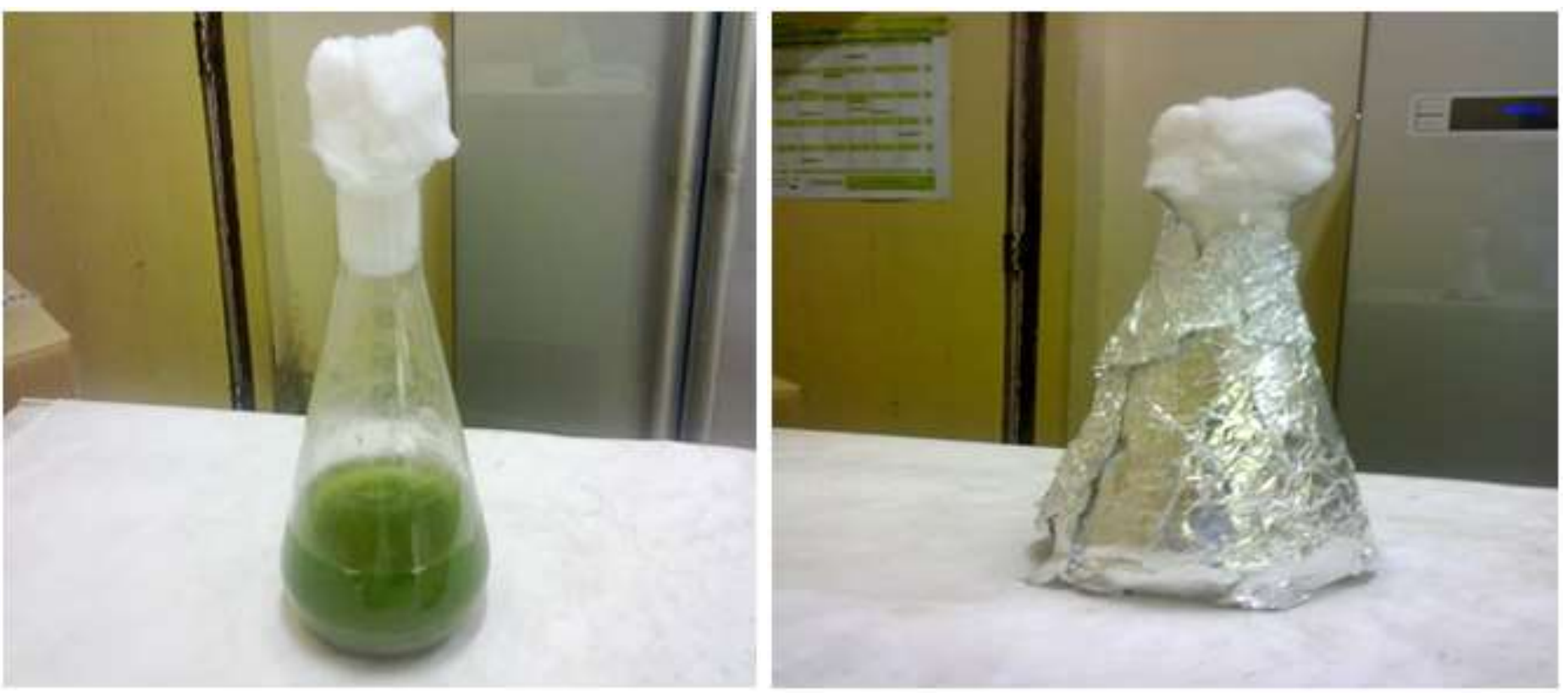

Fig.2. Methodology for heterotrophic growth

Transferred $160 \mathrm{~mL}$ of feed into $5250 \mathrm{~mL}$ flasks (control and 4 flasks with treatment) after that inoculated $20 \mathrm{ml}$ wet biomass of micro algae and culturing for 16 days in heterotrophic conditions.

\section{$\mathrm{CO}_{2}$ Bioremediation by microalgae in} Photobioreactors

Microalgae have the potential to recycle and bioremediate $\mathrm{CO} 2$ and also produce chemical energy in the form of biomass. The potential production of renewable energy and high value products (i.e. carotenoid, antioxidants and polyunsaturated fatty acids) make large scale microalgal cultivation an attractive application. Initially, feed was analyzed before inoculation of microalgae. Nutrients removal is observed by analyzing the nitrates, phosphates and carbohydrates in the feed in alternative days. Feed Samples are collected at five intervals in 16 days cycle for COD removal tests.[3]

\section{Biomass Study}

Algae biomass density and carbohydrate accumulation in algae are also analyzed at alternative days. Lipid accumulation in the algae analyzed in the interval for 4 days in the flasks 1, 2, 3 and 4 respectively.

\section{Lipid Analysis by Sonication}

For knowing lipid content at different time intervals Microalgae Biomass collected from culture flasks and dried under sunlight. Dried biomass grinded and beaded in different solvents. $(2: 1 \mathrm{v} / \mathrm{v}$ Ratio chloroform to methanol for extracting total Lipids) (n Hexane for extracting neutral Lipids).[4]

\section{Gravimetric estimation}

Solvents with dissolved lipids are centrifuged and transferred to aromatic vials and solvent is evaporated.

Through gravimetric estimation lipid percentage in microalgae is estimated. Taken $2 \mathrm{ml}$ vials loaded with $10 \mathrm{mg}$ of dried algal biomass loaded along with $2 \mathrm{ml}$ solvent and Kept for sonication in the Dark Heterotrophic conditions for (16 days) Photo Heterotrophic conditions for (16 days) Photo heterotrophic for (8 days) + Dark heterotrophic for $(8$ days). The extraction was repeated three times, and all the extracts were combined into a pre-weighed glass tube, and then dried under nitrogen protection.

\section{Result and Discussion}

\section{$\mathrm{CO}_{2}$ Bioremediation by microalgae in Photobioreactors}

The addition of $\mathrm{CO} 2$ is required for the autotrophic growth of microalgae, and is considered to be positively related to the efficiency of photosynthesis, with the synthesis of carbohydrates as the end product. A sufficient supply of $\mathrm{CO} 2$ is thus one of the key factors influencing the accumulation of carbohydrate in microalgae. Some studies found that carbohydrate accumulation in microalgae is improved by increasing the percentage of $\mathrm{CO} 2$ in the inlet gas $[4,8]$. For 
example, Xia and Gao [4] pointed out that increasing dissolved $\mathrm{CO} 2$ concentration from 3 to $186 \_\mathrm{mol} / \mathrm{L}$ in the cultivation of $\mathrm{C}$. pyrenoidosa and $\mathrm{C}$. reinhardtii could elevate the carbohydrate content from 9.30 to $21.0 \%$ and 3.19 to $7.40 \%(\mathrm{w} / \mathrm{w})$, respectively. However, many researchers believe that increasing the $\mathrm{CO} 2$ concentration not only provides more carbon source for photosynthesis to promote microalgae growth, but also induces the synthesis of relevant proteins, which may influence the cell physiology. In some microalgal species, increases in the $\mathrm{CO} 2$ concentration result in an increase in the protein content, but a decrease or no obvious change in the carbohydrate content [7, 9]. However, under nitrogen starvation conditions and with an adequate supply of $\mathrm{CO} 2$ and light energy, the protein content in microalgae can be consumed as a nitrogen source, and the carbohydrate content may increase significantly during this process. Therefore, suitable addition of $\mathrm{CO} 2$ is a key step to improving the autotrophic growth of microalgal cells (i.e., improving biomass productivity and protein content), although it may not directly enhance carbohydrate accumulation in microalgae, unless appropriate stress conditions are employed.

\section{Biomass Study}

Fuels made from living biomass rather than organisms fossilised some 200 million years ago have attracted worldwide attention - and suspicion. While development continues apace, first and second generation fuels based on terrestrial plants are controversial because they require cultivation resources that could otherwise be used for growing food. Structural carbohydrates can constitute a large fraction of the dry weight of algal biomass and thus accurate identification and quantification is important for summative mass closure. The comparison was based on the resolution and quantification of monosaccharides obtained after analytical hydrolysis of algal biomass with $\mathrm{H}_{2} \mathrm{SO}_{4}$. [11,12]

\section{Gravimetric estimation}

These valuable chromatography tools have allowed us to characterize the monosaccharides released after sulfuric acid hydrolysis of three types of algae. Further work in structural carbohydrate release, either by chemical or enzymatic hydrolysis, will shed light on not only the total carbohydrate content in algae, but also the understanding of the structure of algal polymeric carbohydrates. This knowledge can be applied to conversion and fermentation experimental work as well as guide research into cell wall degradation procedures to aid with simultaneous oil extraction and release of soluble sugars. A linear correlation between chlorophyll and ammonium concentration in the culture was observed for the experiments at $20^{\circ} \mathrm{C}$ and $25^{\circ} \mathrm{C}$ (Fig. $3 \mathrm{C})$. The pattern suggests that the consumption of this compound was associated with microalgal growth, nevertheless, ammonium may have been also depleted by abiotic phenomena such as stripping related to the high $\mathrm{pH}$ levels induced by the photosynthetic activity (see later) and respired by nitrifying bacteria.[13] The carbohydrate production ability of microalgae varies from species to species. However, as mentioned earlier, the production of microalgal carbohydrates can be significantly improved when appropriate cultivation conditions are applied. Summarizes the performance of the biomass and carbohydrates production of various microalgae species according to recent reports in the literature, showing that the microalgal carbohydrate productivity varies in a wide range from 0.021 to 0.687 $\mathrm{g} / \mathrm{L} / \mathrm{d}$. Our recent work showed that C. vulgaris FSP-E can achieve the highest carbohydrates productivity of $0.687 \mathrm{~g} / \mathrm{L} / \mathrm{d}$ due to a high biomass productivity of 1.363 $\mathrm{g} / \mathrm{L} / \mathrm{d}$. Information concerning the composition of carbohydrates produced by microalgae is also crucial for future applications of microalgae feedstock. However, this information is currently very limited, as only two Chlorella strains. 
Fig 3. OPTIMIZING GROWTH PHASE (Cycle 1) :-Biomass studies

(A) algae biomass density(A)

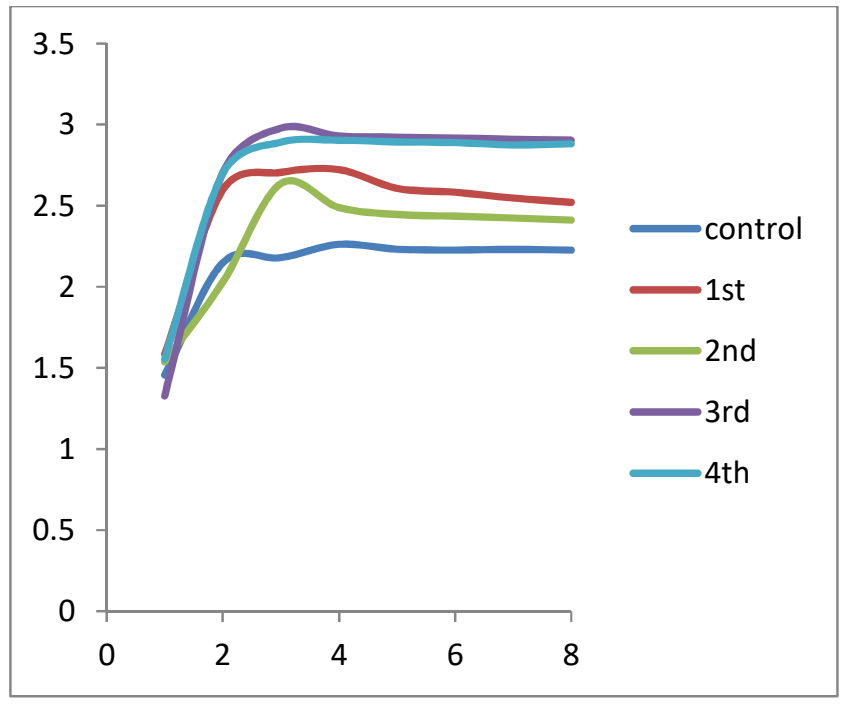

(B) Internal carbohydrates(mg/L)

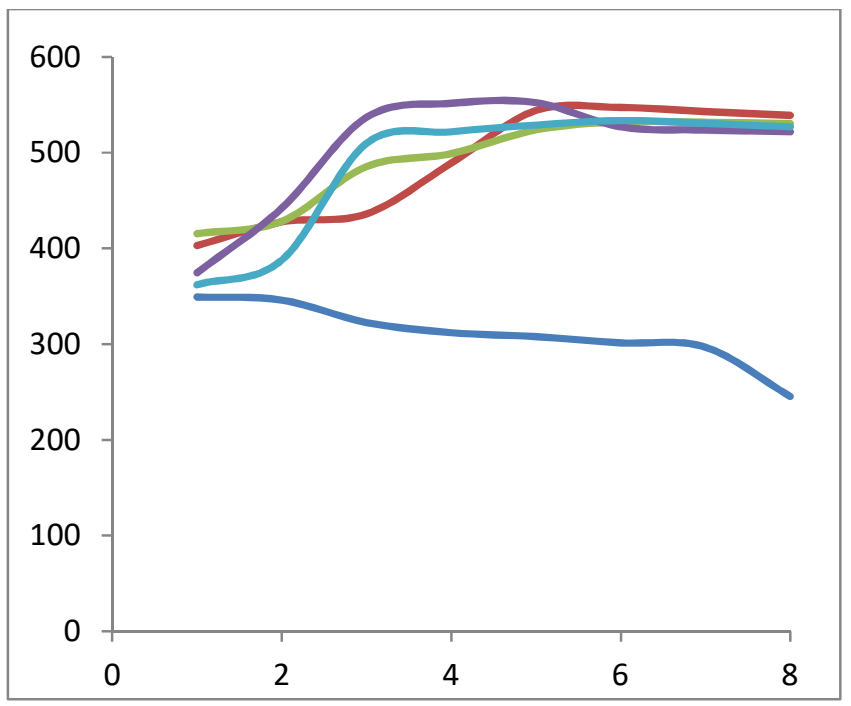

\section{OPTIMIZING GROWTH PHASE (Cycle 1) :-Biomass studies (C) Chlorophyll content(mg/L)}

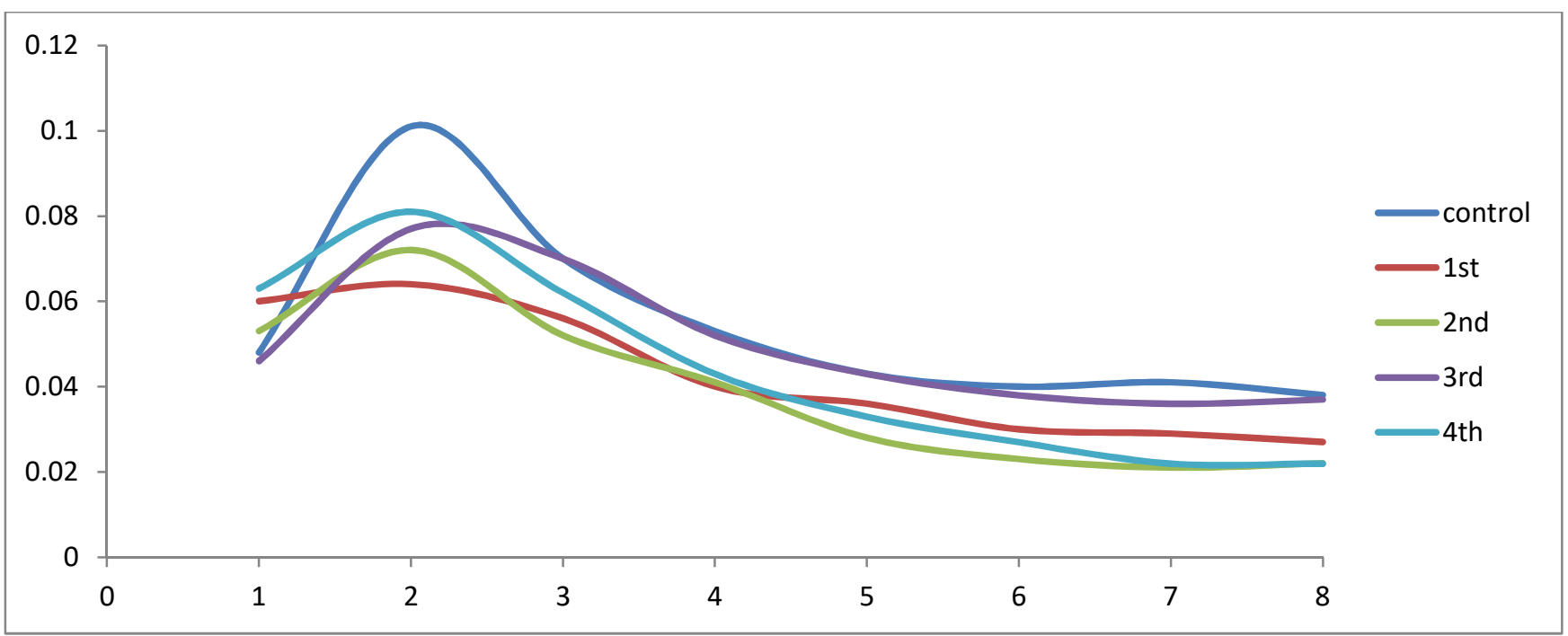

$\mathrm{NH}_{4}{ }^{+}$removal rates in all the cultures showed in the graph at higher temperatures increased and longer photoperiods $\mathrm{PO}_{4}{ }^{3-}$ and $\mathrm{NH}_{4}{ }^{+}$were not removed.[7] This is in agreement with the absence of biological activity in those cultures. Neither biomass production nor nitrification was observed in these conditions (Fig. 4 (A \& B). When correlating the concentration of the residual phosphate in the medium with the chlorophyll concentration of the culture (algal biomass), the correlation coefficients were low (Fig. 4B). This suggested that factors other than assimilation could be at play. Su et al. observed phosphate removal efficacies ranging from 56 to $73 \%$ in batch cultures ( 8 days of incubation) with a low nitrogen/phosphorus ratio $(\mathrm{N} / \mathrm{P}=3: 1)$. This low level of phosphate depletion was attributed to nitrogen limitation encountered by the cultures. The variation in chlorophyll and phosphate concentrations over time were tested at two temperatures and photoperiods tested (Fig. 3 C). During the first $50 \mathrm{~h}$ of the experiment the phosphate concentration fell significantly. At $15{ }^{\circ} \mathrm{C}$, the algae could have accumulated phosphate even though during this period the algal biomass remained approximately constant (Fig. $3 \mathrm{~A}$ and $\mathrm{b}$ ). The fall in the phosphate concentration may be due to adsorption on the cell surface, which has been previously reported to contribute significantly to phosphorus removal from wastewater. At the beginning of the experiment, the magnitude of phosphorus removal was not affected by temperature or photoperiod. This observation could also be explained by adsorption. 
Phosphate removal and biomass production correlated well from $50 \mathrm{~h}$ incubation onwards (Fig. 4 B). From approximately $100 \mathrm{~h}(168 \mathrm{~h}$ for the culture B15-12) onwards the $\mathrm{pH}$ of the cultures rose above 9.5. At this elevated $\mathrm{pH}$, the chemical precipitation of phosphorus is possible and in every graph it can be seen that complete phosphate removal occurs at this $\mathrm{pH}$. No phosphate removal was detected for cultures in darkness where $\mathrm{pH}$ values were between 7.8 and 8.3 all along the incubation period.

Fig 4. OPTIMIZING GROWTH PHASE (Cycle 1) :-Bioremediation studies

(A) Nitrates removal $(\mathrm{mg} / \mathrm{L})$

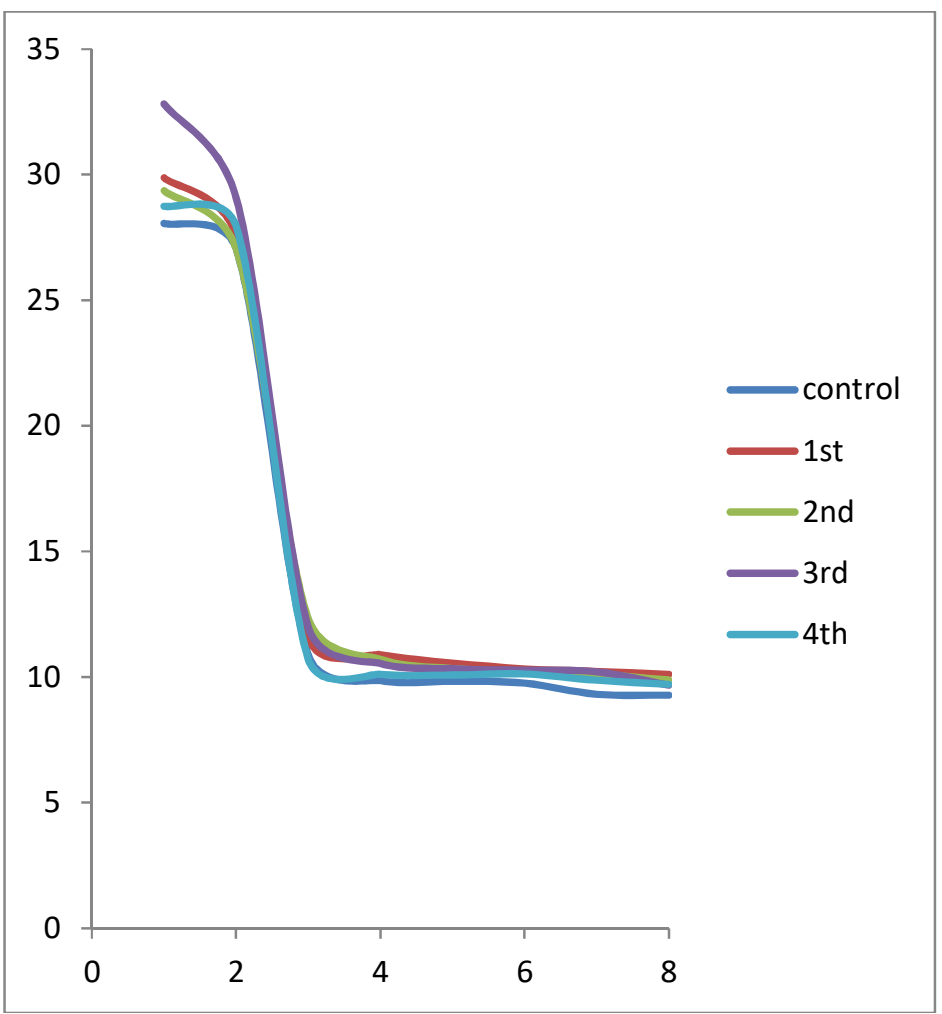

(B)Phosphates removal (mg/L)

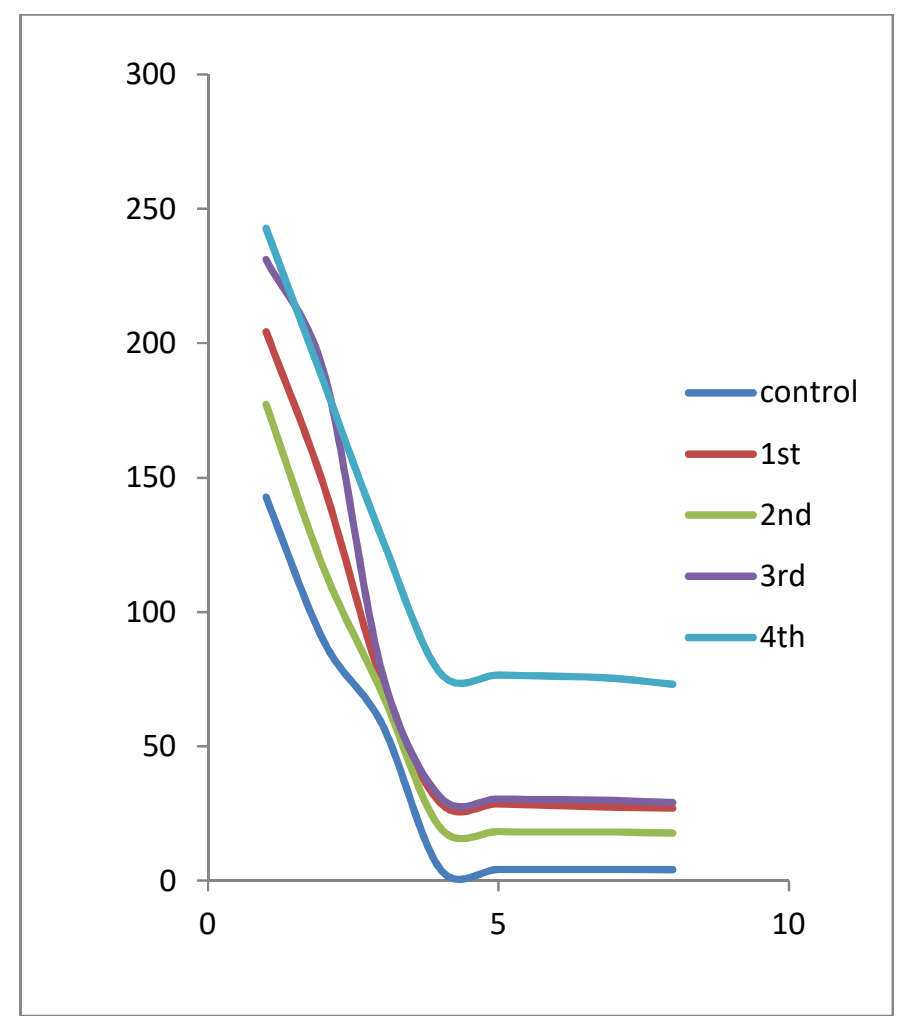


(C) Carbohydrates removal (mg/L)

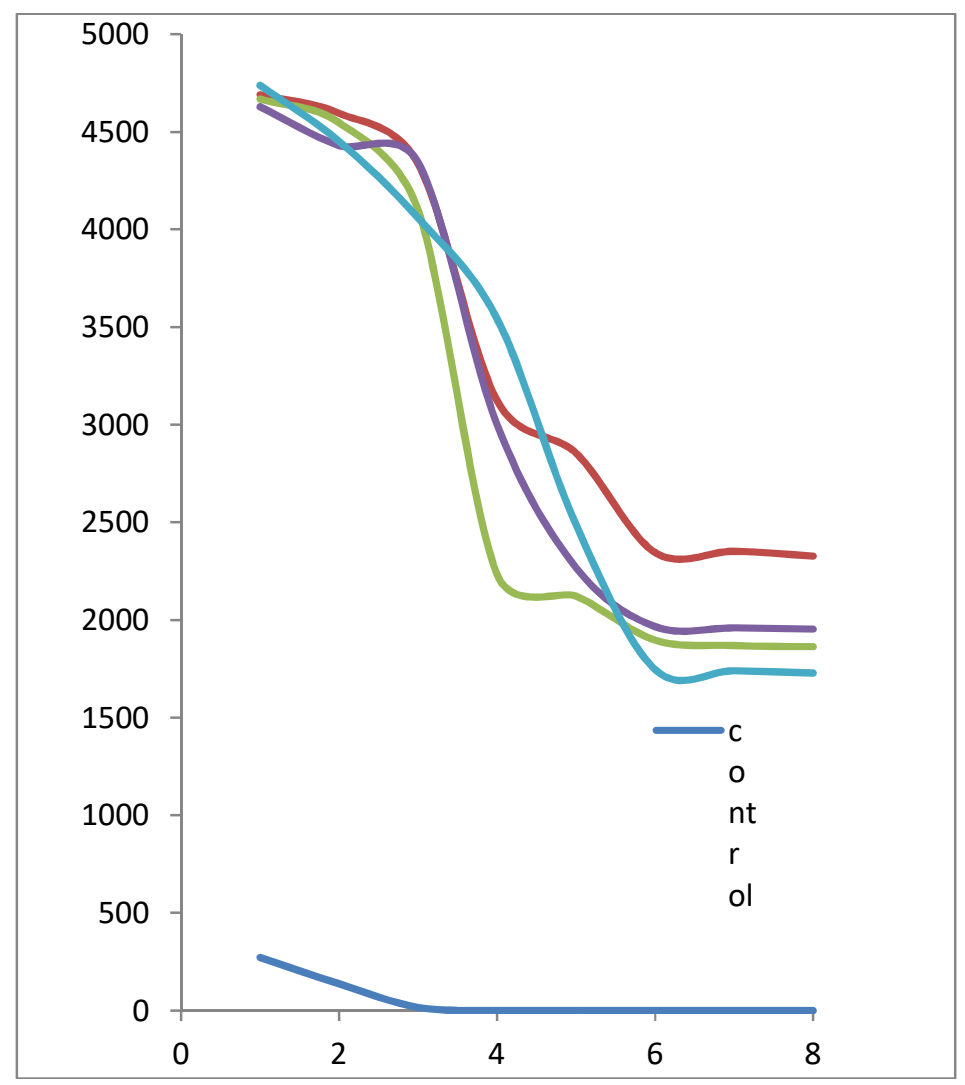

(D) COD removal $(\mathrm{mg} / \mathrm{L})$

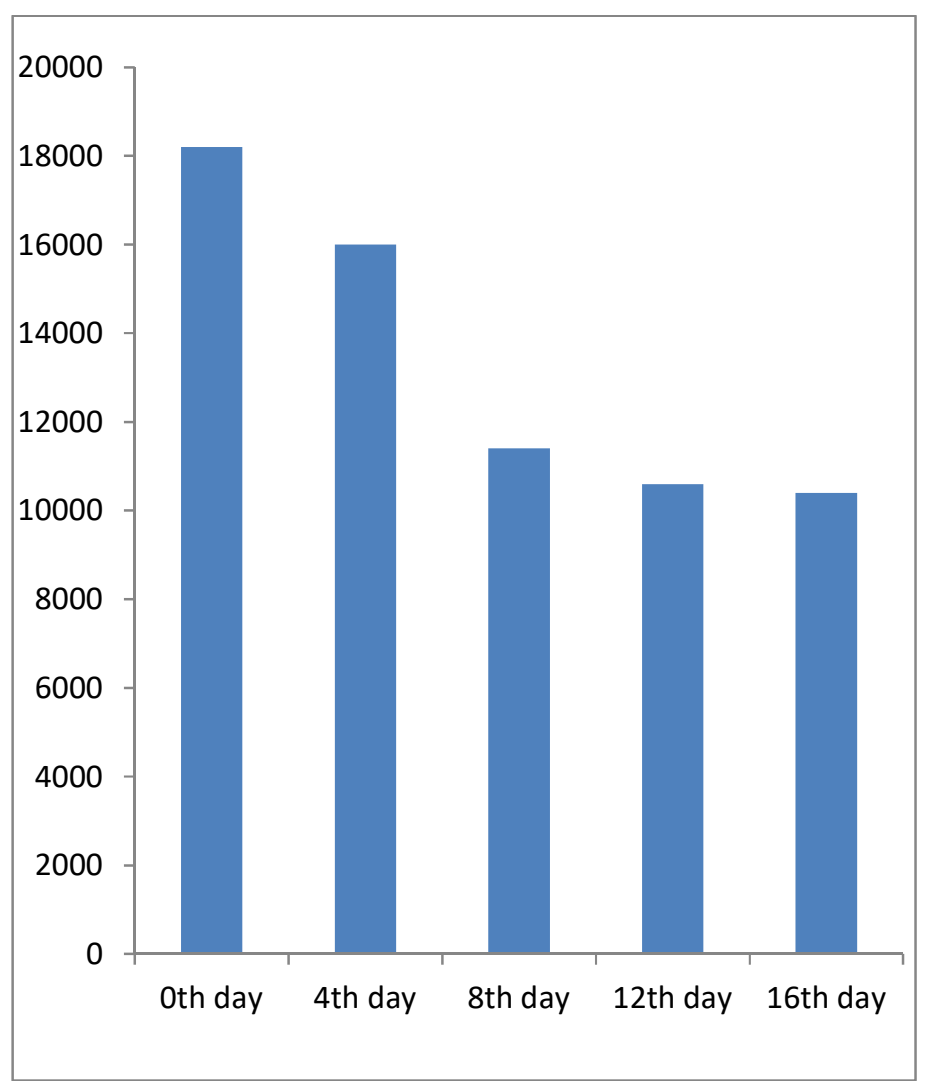

\section{Lipid Analysis by Sonication}

Lipid extracted microalgal biomass residues (LMBRs) using conventional anaerobic activated sludge fermentation technology, generating a new concept for use in biorefineries. Yang et al. [15] showed that the highest biohydrogen yield of $45.54 \mathrm{~mL} / \mathrm{g}-\mathrm{VS}$ was achieved from LMBRs that underwent thermo-alkaline pretreatment at $100{ }^{\circ} \mathrm{C}$, with this being approximately three-fold higher than the yield from untreated LMBRs. In addition, there has recently been growing research attention focused on biogas production from microalgae [16]. However, it is worth noting that the anaerobic digestion process cannot be economically competitive unless it is targeted to treat the microalgal residues remaining after the production of high grade biofuels, such as bioethanol and biobutanol, or unless it is integrated into a microalgae biorefinery [2].

Fig 5. Lipid Analysis

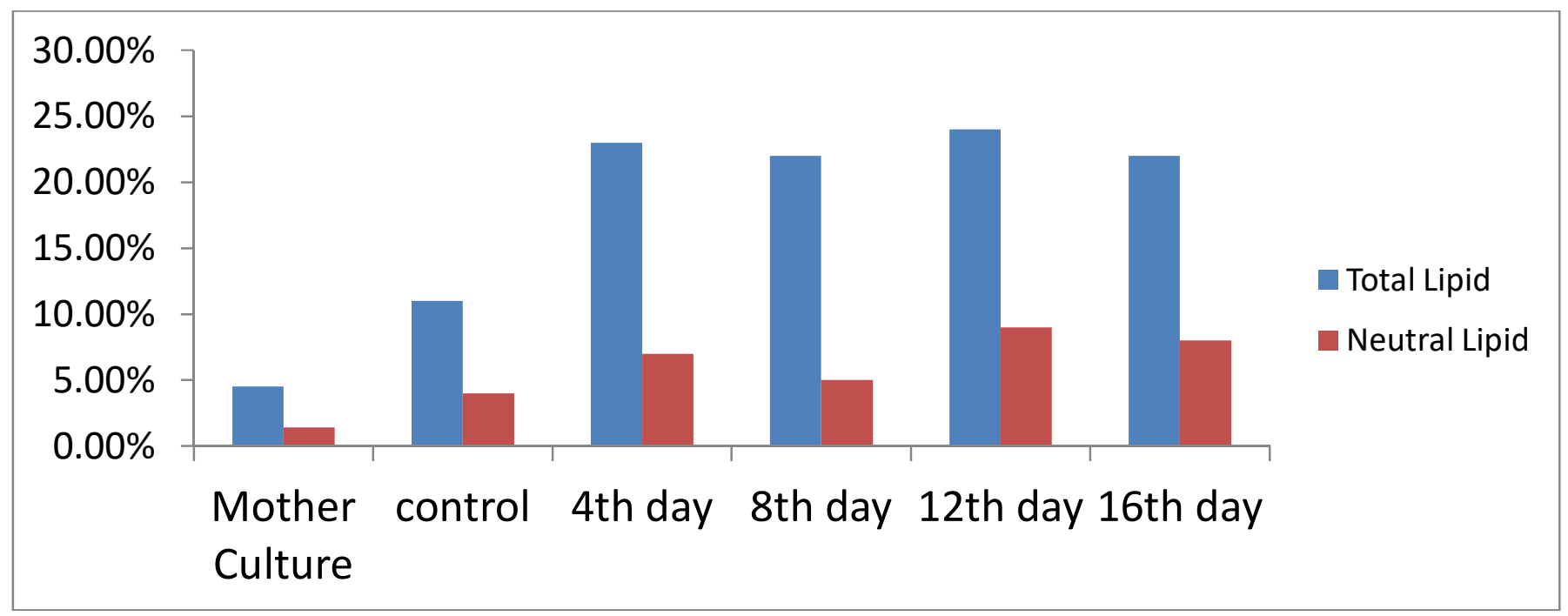


Table 2. Analysis of Lipids

\begin{tabular}{|l|l|l|}
\hline Time Interval & Total Lipid & Neutral Lipid \\
\hline Mother Culture & $4.5 \%$ & $1.4 \%$ \\
\hline control & $11 \%$ & $4 \%$ \\
\hline $4^{\text {th }}$ day & $23 \%$ & $7 \%$ \\
\hline $\mathbf{8}^{\text {th }}$ day & $22 \%$ & $5 \%$ \\
\hline $\mathbf{1 2}^{\text {th }}$ day & $24 \%$ & $9 \%$ \\
\hline $\mathbf{1 6}^{\text {th }}$ day & $22 \%$ & $8 \%$ \\
\hline
\end{tabular}

Under unfavorable and stress conditions microalgae reprogram its pathway towards formation and accumulation of neutral lipids. Heterotrophic growth consumes simple, cheap, and available carbon sources (glucose, acetate, glycerol) that are commonly used by fermentation industries for other aims, it is predicted that adoption of this approach is an easy, uncomplicated task.

\section{References}

1) Chun-Yen Chen, Xin-Qing Zhao, Hong-Wei Yen, Shih-Hsin Ho, Chieh-Lun Cheng,Duu-Jong Lee, Feng-Wu Bai, Jo-Shu Chang. Microalgae-based carbohydrates for biofuel production. Biochemical engineering Journal 78 (2013) 1- 10.

2) J.H. Mussgnug, V. Klassen, A. Schluter, O. Kruse, Microalgae as substrates for fermentative

a. biogas production in a combined biorefinery concept, J. Biotechnol.150 (2010) 51-56.

3) J.R. Xia, K.S. Gao, Impacts of elevated $\mathrm{CO} 2$ concentration on biochemical composition, carbonic anhydrase, and nitrate reductase activity of freshwater green algae, J. Integr. Plant Biol. 47 (2005) 668-675.

4) Kalpesh K. Sharma, Holger Schuhmann and Peer M. Schenk "High Lipid Induction in Microalgae for Biodiesel Production" Energies 2012, 5, 15321553 ;

5) Michael B. Johnson and Zhiyou Wen "Production of Biodiesel Fuel from the Microalga Schizochytrium limacinum by Direct Transesterification of Algal Biomass Energy Fuels 2009, 23, 5179-5183

6) Octavio Perez-Garcia "Heterotrophic cultures of microalgae: Metabolism and potential products" water research 45 ( 2011 ).

7) M. Giordano, Interactions between $\mathrm{C}$ and $\mathrm{N}$ metabolism in Dunaliella salina cells cultured at elevated $\mathrm{CO} 2$ and high $\mathrm{N}$ concentrations, J. Integr. Plant Biol.158 (2001) 577-581.

8) M.R. Brown, S.W. Jeffrey, J.K. Volkman, G.A. Dunstan, Nutritional properties of microalgae for mariculture, Aquaculture 151 (1997) 315-331.
9) Srikanth Reddy Medipally,1 Fatimah Md. Yusoff,1,2 Sanjoy Banerjee,1 andM. Shariff1,3 Microalgae as Sustainable Renewable Energy Feedstock for Biofuel Production BioMed Research International Volume 2015, Article ID 519513, 13 pages http://dx.doi.org/10.1155/2015/519513

10) Tahani S. Gendy *, Seham A. El-Temtamy. Commercialization potential aspects of microalgae for biofuel production: An overview. Egyptian Journal of Petroleum (2013) 22, 43-51

11) Xiaobin Wen1, Kui Du1,2, Zhongjie Wang1, Xinan Peng1,2, Liming Luo1,3, Huanping Tao1, Yan Xu1,2, Dan Zhang1, Yahong Geng1 and Yeguang Li1*.Effective cultivation of microalgae for biofuel production: a pilot-scale evaluation of a novel oleaginous microalga Graesiella sp. WBG-1. Wen et al. Biotechnol Biofuels (2016) 9:123 DOI 10.1186/s13068-016-0541-y.

12) Xuefeng Lu1 "A Perspective: Photosynthetic Production of Fatty Acid-Based Biofuels in Genetically Engineered Cyanobacteria"

13) Yanqun Li \& Mark Horsman \& Bei Wang "Effects of nitrogen sources on cell growth and lipid accumulation of green alga Neochloris oleoabundans Yanqun Li \& Mark Horsman \& Bei Wang“Appl Microbiol Biotechnol (2008) 81:629636

14) Z.M. Yang, R.B. Guo, X.H. Xu, X.L. Fan, X.P. Li, Enhanced hydrogen production from lipid-extracted microalgal biomass residues through pretreatment, Int. J.Hydrogen Energy 35 (2010) 9618-9623.

15) P. Collet, A. Helias, L. Lardon, M. Ras, R.A. Goy, J.P. Steyer, Life-cycle assessment of microalgae culture coupled to biogas production, Bioresour. Technol. $\quad 102 \quad$ (2011) 207-214. 